\section{Profiting from biodiversity}

SIR-Many people see biodiversity as an expensive luxury which we either have a duty to pay for or simply cannot afford. A glance at almost any first-world dinner table, covered with food species from around the world, shows this is nonsense - biodiversity is an immense economic resource. Profitable and sustainable economic exploitation of biodiversity is perhaps the best way to ensure its maintenance. We recently attended a conference at Roros, central Norway in which Dan Janzen described a programme to turn Costa Rica into a country which profitably manages and exploits its biodiversity.

The first step is to take inventory. Janzen spends six months a year training 'parataxonomists' - former farmers, other rural workers and students - to provide the raw material for Costa Rica's National Biodiversity Institute. The institute itself, a private, non-profit making, public-service organization, integrates information on resources from the flora and fauna of the national parks which constitute more than a quarter of Costa Rica's land surface area with a view to exploiting it economically.

After training, the parataxonomists leave for designated patches of park to compile collections of local organisms. The collections are housed and handled at the institute by in-house curators. As the samples are identified to finer taxonomic levels, the curators call on expertise from abroad. The inventory already includes at least one potential moneyspinner. Janzen showed us a plant which, he claims, is rich in antibiotics. He refused to tell us the plant's natural location, as the intention is to develop its potential so that the economic benefits are channelled back into the preservation of Costa Rican biodiversity.

Janzen estimates that the contents of the major national parks should be catalogued within a decade: the first two classes of parataxonomists, comprising 33 students, are now working in the country's eight conservation areas. A local and regional infrastructure is now in place for the later stages of Costa Rica's biodiversity programme, which will include more fundamental research as well as commercial exploitation.

Who is paying for all this? The Costa Rican government, although an integral part of the enterprise, is not wealthy. Initially, a series of institutions, including private foundations, private donors and national governments, provided $\$ 2.53$ million for the first two years of operation. In the long term, one scheme is that initial capitalization of $\$ 30$ million would purchase $\$ 120$ million of Costa Rican international debt. The Costa Rican government would then buy the $\$ 120$ million by paying $\$ 96$ million to a biodiversity institute trust fund, which would pay $3 \%$ annual interest in local currency.

The National Biodiversity Institute represents a different attitude towards third-world biodiversity than that which characterizes the developed countries, where biodiversity tends to be viewed as a common heritage of mankind. This belief must be eliminated or else biodiversity will suffer the tragedy of the commons, with everyone trying to make a quick profit but unwilling to invest in sustaining the resource. If Costa Rica benefits economically from its biodiversity, then Costa Ricans will have an economic interest in the sustainable exploitation of their own natural resources. If successful, the institute will provide a model for similar schemes elsewhere.

SEAN NEE

Department of Zoology,

PaUl H. HaRVEY

University of Oxford,

South Parks Road,

Oxford OX1 3PS, UK

\section{Ellipses and ellipsis}

SIR-Klaczko and Bitner-Mathe in their Scientific Correspondence ${ }^{1}$ fit ellipses to wing outlines of Drosophila. The fit is impressive and they suggest that ellipse shape can be used to quantify wing shape.

But the same ellipse can exactly fit outlines of very different shapes (Fig. 1). Such ambiguity arises when outlines are not closed, so that only part of the ellipse need fit. Klaczko and Bitner-Mathe digitize the wing tip and front margin (from A to D in Fig. 2). Although open, this portion is evidently sufficient to fit an ellipse representative of the entire, almost closed, wing periphery. But we may be interested in the open outline $\mathrm{AD}$ per se (developmentally, this and the hind margin belong to different compartments). In that case, to avoid equating dissimilar outlines requires two further parameters specifying which arc of the ellipse is used - for instance parameters $\theta$ and $\cos ^{-1}$ $(c / d)$ in Fig. 2 (top).

Unfortunately, although in combina-

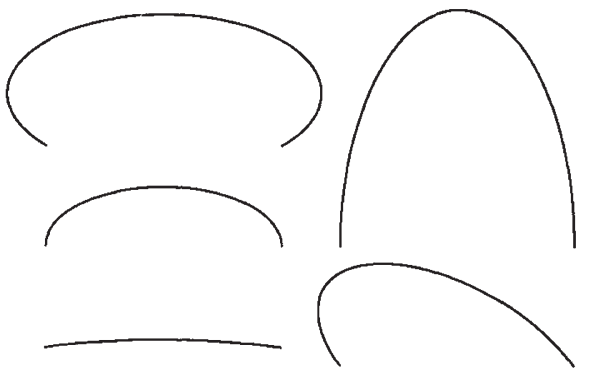

FIG. 1 Five arcs from the same-shaped ellipse.

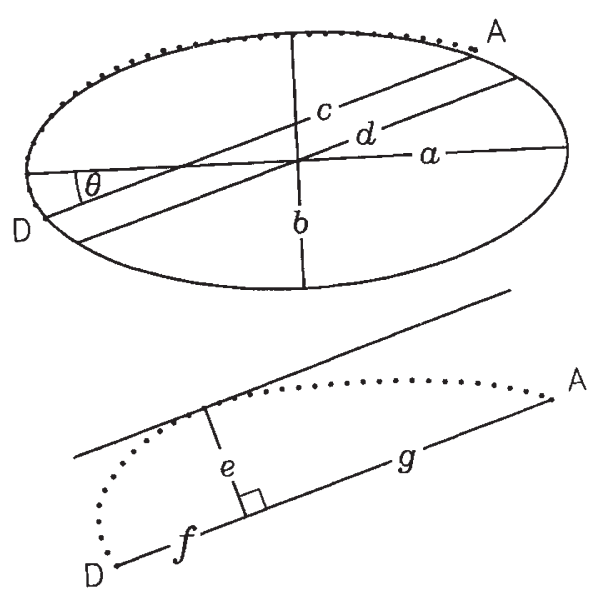

FIG. 2 Top, The dotted line AD represents the digitized wing outline, to which Klaczko and Bitner-Mathe fitted the ellipse. The line of length $c$ cuts off the ellipse arc adjacent to the outline. Bottom, A simpler geometrical construction.

tion ellipse shape and these parameters specify fully the shape of an ellipse arc, each parameter remains difficult to interpret when the others are changing. Unless parameters can be chosen to match observed limits to variation, a change in outline recognizable as a single, simple distortion may affect all ellipse parameters in a confusing, nonlinear way. An ellipse arc geometrically intermediate between another two arcs need not have intermediate parameters; if parameters are intermediate, the arc need not appear geometrically intermediate (Fig. 3). Nor is the panacea to enter all the ellipse parameters in a multivariate analysis: we should

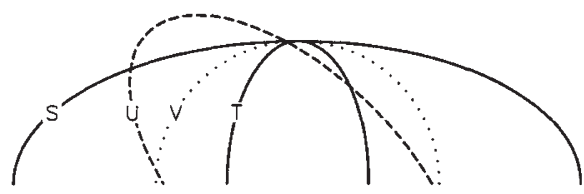

FIG. 3 Ellipses S, T and U share the same value of $b / a$ and $c / d$, and $U$ is intermediate in values of $\sqrt{a b}$ and $\theta$. Ellipse $V$ differs in $b / a$ and so is not intermediate between $S$ and $T$ for this set of parameters.

not expect linear combinations to disentangle trigonometric inter-relationships.

A few-parameter summary of shape may be more comprehensible if based simply on relative distances between homologous landmarks and extremum points (see parameters $\log [e /(f+g)]$ and $\log [g /(f+g)]$ in the bottom part of Fig. 2). Alternatively, as wing venation provides a wealth of unambiguous landmarks, more advanced mathematical tools can be used to analyse shape variation ${ }^{2}$.

Nevertheless, ellipses have been sensibly used to quantify biological shape ${ }^{3}$. Sampson has developed ${ }^{4}$ a fitting algorithm unaffected by the outline's orientation which generates confidence limits summarizing variation between outlines. The technique also allows ellipses to be constrained, for instance to lie parallel to 